\title{
Assessment of Oxidative Stress in Sperm and Semen
}

\author{
Anthony H. Kashou, Rakesh Sharma, and Ashok Agarwal
}

\begin{abstract}
The chemiluminescence method is the most commonly employed technique as a direct measurement of reactive oxygen species (ROS) generation by spermatozoa. This assay is capable of quantifying both intracellular and extracellular ROS. Moreover, the use of various probes allows for differentiation between superoxide and hydrogen peroxide production by spermatozoa. When the total antioxidant reserves are overwhelmed by excessive production of ROS, it results in oxidative stress. Therefore correct measurement of both ROS and total antioxidant capacity (TAC) is essential in the assessment of oxidative stress in sperm and semen. This chapter describes the methodological approach for measuring seminal oxidative stress through the use of chemiluminescence assay for accurate measurement of ROS and the colorimetric assay for measurement of TAC.
\end{abstract}

Key words: Chemiluminescence, Seminal plasma, Spermatozoa, Antioxidant capacity, Oxidative stress

\section{Introduction}

\subsection{Reactive Oxygen Species}

Male infertility represents a vexing problem of great magnitude. Reactive oxygen species (ROS) affect and influence spermatozoa in their local environments. Any imbalance between ROS production and the biological system's ability to scavenge these reactive intermediates by antioxidants (both enzymatic and nonenzymatic) results in a condition known as oxidative stress (OS). Disturbances in the normal redox state can have deleterious effects on the body. OS has been implicated in several diseases, along with impaired sperm parameters. Moreover, OS levels have been shown to correlate with reduced fertility (1).

Therefore, accurate ROS measurement provides a vital tool in the initial evaluation and follow-up of infertile male patients. 
Establishing reference values may help in identifying the potential causes of reduced fertility and developing strategies to reduce OS and improve sperm quality. ROS can be produced both by peroxidase-positive leukocytes and defective morphologically abnormal spermatozoa with excessive residual cytoplasm present in the midpiece area. Common ROS are superoxide anion $\left(\mathrm{O} 2^{\cdot-}\right)$, hydroxyl radical $\left(\mathrm{OH}^{\bullet}\right)$, and the strong oxidizer hydrogen peroxide $\left(\mathrm{H}_{2} \mathrm{O}_{2}\right)$. ROS or their oxidized products can be measured both by direct assays such as chemiluminescence, nitroblue tetrazolium test, cytochrome $c$ reduction, flow cytometry, electron spin resonance, and xylenol orange-based assay. Indirect methods include measurement by myeloperoxidase test, measurement of redox potential, lipid peroxidation levels, chemokines, antioxidants, and antioxidant enzymes and measuring levels of DNA damage $(2,3)$.

Two probes may be used with the chemiluminescence assay: luminol and lucigenin. A luminol-mediated chemiluminescence signal in spermatozoa occurs when luminol oxidizes at the acrosomal level. Luminol reacts with a variety of ROS and allows both intracellular and extracellular ROS to be measured. Lucigenin is more specific for superoxide anions released extracellularly $(2,3)$.

The luminol assay is more advantageous for a number of reasons. It can measure $\mathrm{H}_{2} \mathrm{O}_{2}, \mathrm{O}_{2}{ }^{--}$, and $\mathrm{OH}^{--}$levels. However, it cannot distinguish these oxidants from one another (4). In addition, luminol is easy to use and can measure the global level of ROS under physiological conditions. Since the assay can measure both extracellular and intracellular ROS, it has a high sensitivity (4). Multiple studies have correlated high chemiluminescent signals using luminol as a probe with adverse effects on sperm function.

This chapter aims to provide a step-by-step approach in the use of chemiluminescence assay utilizing luminol as a probe for the assessment of ROS in sperm and semen. The instrument used to measure the light intensity resulting from chemiluminescence reaction is called luminometer. These can be single tube, multiple tube, or plate luminometers. Depending on the nature of the manner in which the signal input is measured they can be either the photoncounting luminometers that count individual photons or the direct current luminometers that measure electric current that is proportional to the photon flux passing through the photomultiplier tube. The results can be expressed as relative units (RLU), counted photons per minute (cpm), or millivolts/second. The method described below utilizes a multiple tube luminometer, which is a photoncounting instrument that covers a spectral range from 390 to $620 \mathrm{~nm}$. ROS can be measured in either neat or unprocessed liquefied seminal ejaculate, washed sperm, or sperm prepared by swim up or by density gradient separation.

1.2. Total Antioxidant Capacity
Living organisms have developed a complex antioxidant system to counteract the effects of ROS and reduce damage. The antioxidant system of living organisms includes enzymes such as superoxide 
dismutase, catalase, and glutathione peroxidase; macromolecules such as albumin, ceruloplasmin, and ferritin; and an array of small molecules, including ascorbic acid, $\alpha$-tocopherol, $\beta$-carotene, reduced glutathione, uric acid, and bilirubin. The sum of endogenous and food-derived antioxidants represents the total antioxidant activity of the extracellular fluid. Thus, the overall antioxidant capacity may give more relevant biological information compared to that obtained by the measurement of individual components, as it considers the cumulative effect of all antioxidants present.

A simple assay kit is available from Cayman Chemicals (antioxidant assay kit, Ann Arbor, MI). We have standardized this assay and use it in our laboratory setting. The assay relies on the ability of antioxidants in the sample to inhibit the oxidation of $2,2^{\prime}$-azinodi-[3-ethylbenzthiazoline sulfonate] (ABTS) to ABTS+ by metmyoglobin. Under the reaction conditions used, the antioxidants in the seminal plasma cause suppression of the absorbance at $750 \mathrm{~nm}$ to a degree that is proportional to their concentration. The capacity of the antioxidants present in the sample to prevent ABTS oxidation is compared with that of the standard-Trolox, a water-soluble tocopherol analogue. Results are reported as micromoles of Trolox equivalent. This assay measures the combined antioxidant activities of all its constituents including vitamins, proteins, lipids, glutathione, uric acid, etc.

1.3. Conclusion

Accurate measurement of both ROS and total antioxidant capacity (TAC) is important to determine if oxidative stress is the underlying cause of male infertility. In such conditions, it is important to reduce the ROS levels by eliminating the ROS generation by leukocytes in case of an underlying infection, and antioxidant supplementation to improve TAC may prove to be beneficial.

\section{Materials}

\subsection{Equipment and Consumables for ROS Assay}

1. Disposable polystyrene tubes with caps $(15 \mathrm{~mL})$.

2. Eppendorf pipets $(5,10 \mu \mathrm{L})$.

3. Serological pipets $(1,2,10 \mathrm{~mL})$.

4. Desktop centrifuge.

5. Disposable sperm-counting chamber.

6. Dimethyl sulfoxide (DMSO).

7. Luminol (5-amino-2,3-dihydro-1,4-phthalazinedione).

8. Polystyrene round-bottom tubes $(6 \mathrm{~mL})$.

9. Multiple tube luminometer

10. Dulbecco's Phosphate-Buffered Saline Solution $1 \times(\mathrm{PBS})$. 
2.2. Reagents for ROS Assay

\subsection{Equipment and Materials for TAC Assay}

1. DMSO solution: Provided ready to use (see Note 1).

2. Stock Luminol (100 mM): Weigh out and add $177.09 \mathrm{mg}$ of luminol to $10 \mathrm{~mL}$ of DMSO in a polystyrene tube (see Notes 2 and 3 ).

3. Working Luminol ( $5 \mathrm{mM}$ ): Mix $380 \mu \mathrm{L}$ DMSO with $20 \mu \mathrm{L}$ of prepared luminol stock solution in a foil-covered polystyrene tube (see Note 4 ).

1. Antioxidant assay kit (Cayman Chemical, Ann Arbor, MI).

2. Absorbance Microplate Reader (e.g., BioTek Instruments, Inc., Winooski, VT).

3. Pipettes $(20,200$, and $100 \mu \mathrm{L})$.

4. Pipette tips $(20,200$, and $100 \mu \mathrm{L})$.

5. Multichannel pipettes (eight channel, 30-300 $\mu \mathrm{L}$ ).

6. Aluminum foil.

7. Microfuge tubes.

8. Deionized water.

9. Polystyrene centrifuge tubes (50 and $15 \mathrm{~mL}$ ).

10. Round-bottom tubes $(12 \times 75 \mathrm{~mm})$.

\section{Methods}

\subsection{Specimen Preparation and ROS Measurement}

1. Herein we describe the details of measurement of ROS by chemiluminescence assay. ROS can be measured by the chemiluminescence method using a probe called luminol. Luminol is extremely sensitive and reacts with a variety of ROS at neutral $\mathrm{pH}$. It has the ability to measure both extracellular and intracellular ROS. Free radicals have a very short half-life and are continuously produced. The free radical combines with luminol to produce a light signal that is then converted to an electrical signal (photon) by the instrument called luminometer. The number of free radicals produced is measured as $\mathrm{RLU} / \mathrm{s} / \times 10^{6}$ sperm.

2. Upon collection of semen sample, incubate at $37^{\circ} \mathrm{C}$ for $20 \mathrm{~min}$ to allow for liquefaction. Record initial characteristics, including semen volume, $\mathrm{pH}$, and color, and manually assess sperm count, concentration, and motility. Thereafter, process the semen specimen for ROS measurement as described below (5) (Fig. 1).

3. The following samples/preparations can be used for ROS measurement: neat or unprocessed sample (seminal ejaculate after 


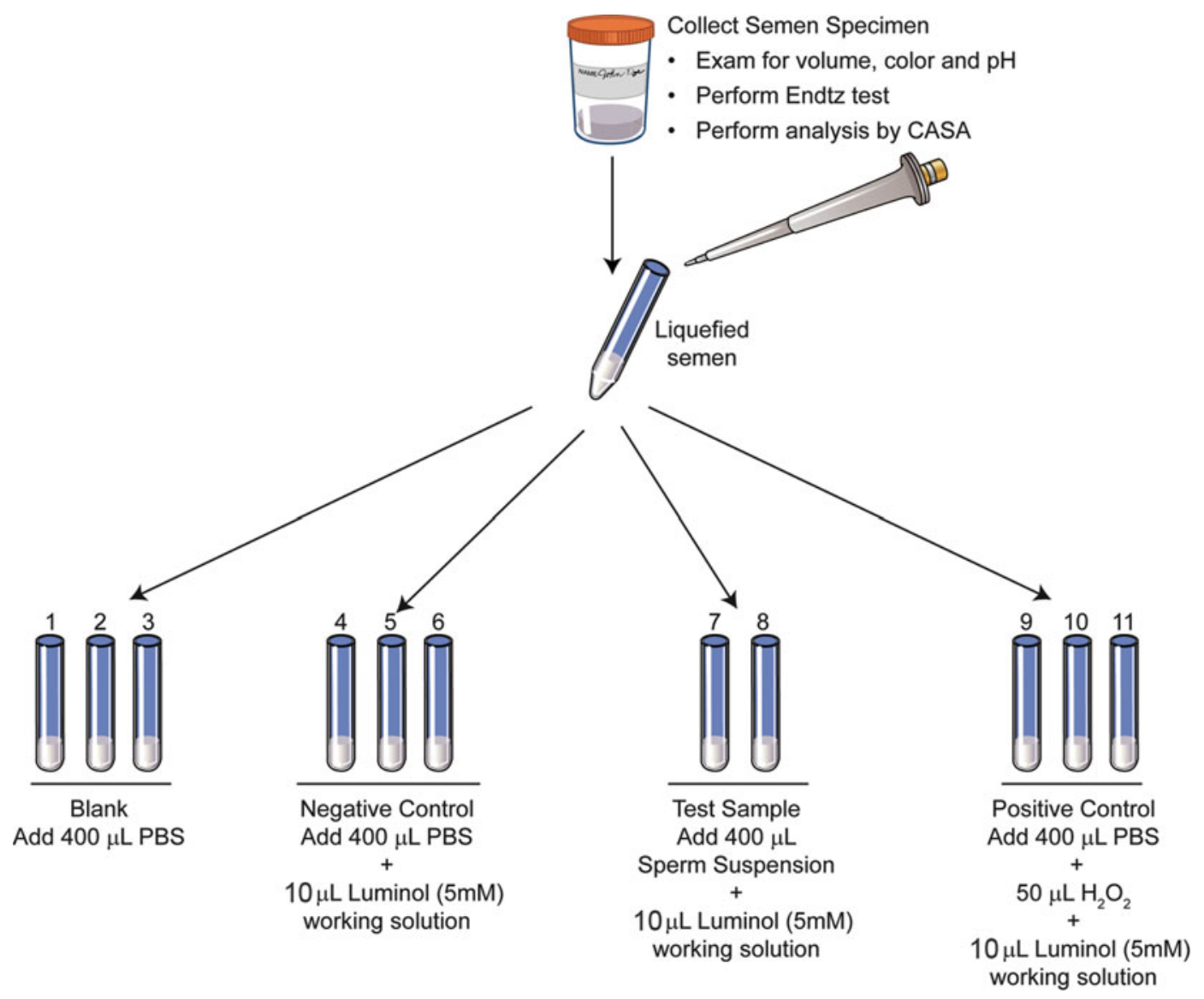

Fig. 1. Workflow for ROS measurement using luminol.

3.2. ROS Measurement with Luminometer (see Fig. 2) liquefaction) (6), washed sample (see Note 5), sample prepared by swim-up method (see Note 6), or sample prepared by density gradient centrifugation (see Note 7 ).

1. Turn on the luminometer and ensure that it is ready for measurements.

2. Enter information in the luminometer including the patient information, number of samples, single measuring time, and data points measured in the integrated mode. Generally, the measurement time is $15 \mathrm{~min}$.

3. Label $6 \mathrm{~mL}$ tubes for blank, negative control, test sample and positive control and add reagents as shown in Table 1 (see Note 8 ). The blank, negative controls and positive controls are run in triplicate, and patient samples are typically run in duplicate, though they can be run in triplicate if ejaculate volume is sufficient. 


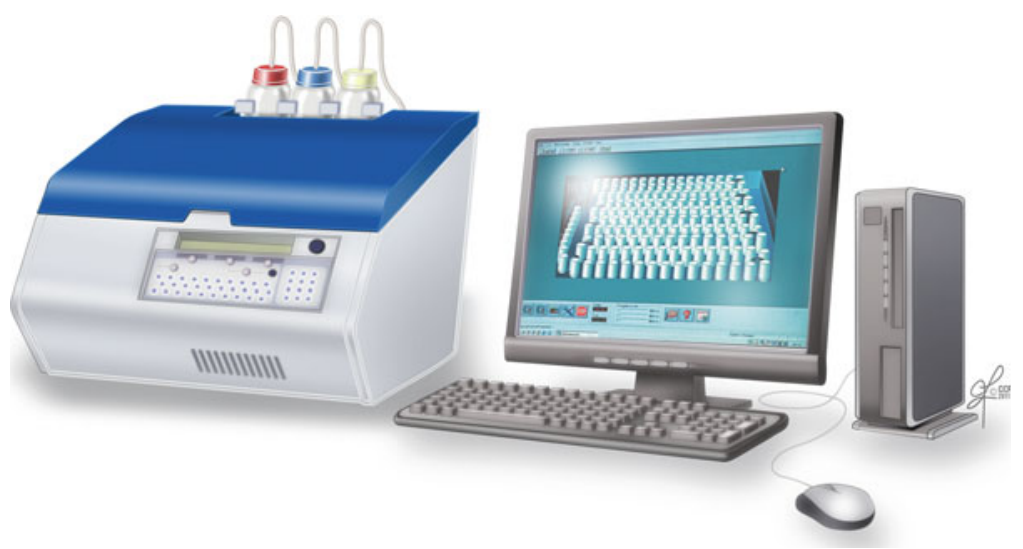

Fig. 2. Illustration of a typical luminometer setup for luminol based ROS analysis. Reprinted with permission from the Cleveland Clinic Center for Medical Art \& Photography @) 2011.

\section{Table 1}

\section{Components and volumes for preparation of the various tubes for measuring ROS}

\begin{tabular}{llllll} 
No. & Labeled tube & $\begin{array}{l}\text { PBS volume } \\
(\mu \mathrm{L})\end{array}$ & $\begin{array}{l}\text { Specimen } \\
\text { volume }(\mu \mathrm{L})\end{array}$ & $\begin{array}{l}\text { Luminol } \\
(\mathbf{5} \mathbf{~ m M})\end{array}$ & $\begin{array}{l}\text { Hydrogen peroxide } \\
(\mathbf{8 - 9} \mathbf{~ M )}\end{array}$ \\
\hline 1 & Blank (tubes S1-S3) & 400 & - & - & - \\
\hline 2 & $\begin{array}{c}\text { Negative control } \\
\text { (tubes S4-S6) }\end{array}$ & 400 & - & $10 \mu \mathrm{L}$ & - \\
\hline 4 & Patient (tubes S7-S8) & - & 400 & $10 \mu \mathrm{L}$ & - \\
\hline 5 & $\begin{array}{c}\text { Positive control } \\
\text { (tubes S9-S11) }\end{array}$ & - & 400 & $10 \mu \mathrm{L}$ & $50 \mu \mathrm{L}$ \\
\hline
\end{tabular}

\subsection{Calculating} Results for the ROS Assay
4. Immediately after adding the probe, initiate measurements. Load the tubes and operate the luminometer according to manufacturer's recommendations.

5. After finishing the measurements, save the data for analysis.

1. Calculate the "average RLU" for Negative control, Samples, and Positive control.

2. Calculate sample ROS by subtracting its average from negative control average: Sample ROS = Average "RLU mean" for sample-Average "RLU mean" for negative control.

3. Correct the sample ROS by dividing it by "Sperm concentration/mL" (see Note 9).

4. Each lab can establish its reference value for ROS by running a large number of samples from healthy and infertile subjects. 


\subsection{Preparation of Assay Reagents for TAC Assay}

In our laboratory, we have established the reference values as follows: Normal range: $<20 \mathrm{RLU} / \mathrm{s} / 10^{6}$ sperm. Critical values: $>20 \mathrm{RLU} / \mathrm{s} / 10^{6}$ sperm.

5. Evaluate replicates to ensure that they are concordant within acceptable limits and ensure that control values are within the normal range (see Note 10).

All reagents should be equilibrated at room temperature before beginning the assay and prepared according to the manufacturer's instructions provided with the assay kit and also available at www. caymanchem.com.

1. Prepare Antioxidant assay buffer $(10 \times)$ (vial \# 1): Dilute $3 \mathrm{~mL}$ of assay buffer concentrate with $27 \mathrm{~mL}$ of HPLC-grade water in a $50 \mathrm{~mL}$ conical tube (see Note 11 ).

2. Prepare chromogen (vial \# 2): Reconstitute the chromogen (containing ABTS) with $6 \mathrm{~mL}$ of water and vortex it. This volume is sufficient for 40 wells (see Note 12).

3. Prepare metmyoglobin (vial \# 3): Reconstitute the lyophilized powder with $600 \mu \mathrm{L}$ of assay buffer and vortex it. Once reconstituted, it is sufficient for 60 wells (see Note 13).

4. Prepare Trolox (vial \# 4): This vial contains the standard Trolox (6-hydroxy-2, 5,7,8-tetramethylchroman-2-carboxylic acid). Reconstitute the lyophilized powder in the bottle with $1 \mathrm{~mL}$ of water and vortex it. This is used to prepare the standard curve (see Note 14).

5. Prepare hydrogen peroxide (vial \# 5): This vial contains $8.82 \mathrm{M}$ solution of hydrogen peroxide. Dilute $10 \mu \mathrm{L}$ of hydrogen peroxide reagent with $990 \mu \mathrm{L}$ of water. Further dilute by removing $20 \mu \mathrm{L}$ and diluting with $3.98 \mathrm{~mL}$ of water to give $441 \mu \mathrm{M}$ working solution (see Note 15).

1. Bring frozen seminal plasma to room temperature and centrifuge in a microfuge at high speed for $5 \mathrm{~min}$. Remove clear seminal plasma and dilute each sample $1: 10(10 \mu \mathrm{L}$ sample $+90 \mu \mathrm{L}$ assay buffer) in a microfuge tube (see Note 16).

2. Use a plate template to note the locations of each sample (run standard and test samples in duplicate (see Note 17)).

3.6. TAC Determination

1. Prepare the standards in seven clean tubes and mark them A-G. Add the amount of reconstituted Trolox and Assay buffer to each tube as shown in Table 2.

2. Add $10 \mu \mathrm{L}$ of Trolox standard (tubes $\mathrm{A}-\mathrm{G}$ ) or sample in duplicate $+10 \mu \mathrm{L}$ of metmyoglobin $+150 \mu \mathrm{L}$ of chromogen per well (see Note 18). 


\section{Table 2}

\section{Preparation of the Trolox standards}

\begin{tabular}{lcll} 
Tube & $\begin{array}{l}\text { Reconstituted Trolox } \\
(\mu \mathrm{L})\end{array}$ & $\begin{array}{l}\text { Assay buffer } \\
(\mu \mathrm{L})\end{array}$ & $\begin{array}{l}\text { Final concentration } \\
(\mathrm{mM} \text { Trolox })\end{array}$ \\
\hline A & 0 & 1,000 & 0 \\
\hline B & 30 & 970 & 0.044 \\
\hline C & 60 & 940 & 0.088 \\
\hline D & 90 & 910 & 0.135 \\
\hline E & 120 & 880 & 0.18 \\
\hline F & 150 & 850 & 0.225 \\
\hline G & 220 & 780 & 0.330 \\
\hline
\end{tabular}

3. Initiate the reaction by adding $40 \mu \mathrm{L}$ of hydrogen peroxide working solution using a multichannel pipette (see Note 19).

4. Cover the plate with the plate cover and incubate on a shaker for $5 \mathrm{~min}$ at room temperature.

5. Remove the cover and read the absorbance at $750 \mathrm{~nm}$ using a plate reader.

3.7. Calculation of TAC Results
1. Calculate the average absorbance of each standard and sample.

2. Calculate the antioxidant concentration of the samples using the equation obtained from the linear regression of the standard curve by substituting the average absorbance values for each sample into the following equation (see Note 20):

Antioxidant $(\mu \mathrm{M})=\frac{\text { Unknown average absorbance }-Y \text { intercept }}{\text { Slope }} \times$ dilution $\times 1,000$

3. Evaluate results against a reference range (see Note 21).

4. Evaluate the ROS-TAC score for each sample (see Note 22).

\section{Notes}

1. Store at room temperature until the expiration date.

2. Cover the tube with aluminum foil to protect the luminol from light.

3. Store stock solution at room temperature in the dark until expiration date. 
4. Prepare working luminol prior to each use.

5. Seminal plasma is removed by washing and resuspending the sample in culture media (7). Measurement is done after liquefied semen specimens are centrifuged at $300 \times g$ for $7 \mathrm{~min}$ and seminal plasma is removed. The sperm pellet is then washed and resuspended in $1 \mathrm{~mL}$ PBS. While this approach allows for the removal of seminal plasma and other dissolved components, all cellular components-round cells, while blood cells, leukocytes, and debris - are still present in the sample.

6. Sperm preparation by the swim-up method: Following liquefaction, mix specimen with sperm wash media using a sterile Pasteur pipette. Centrifuge at $330 \times g$ for $10 \mathrm{~min}$. Aspirate supernatant and resuspend the pellet in $3 \mathrm{~mL}$ of fresh sperm wash media. Transfer the resuspended sample in equal parts to two $15 \mathrm{~mL}$ sterile round-bottom test tubes, and centrifuge at $330 \times g$ for $5 \mathrm{~min}$. Incubate test tubes at a $45^{\circ}$ angle in $5 \% \mathrm{CO}_{2}$ at $37^{\circ} \mathrm{C}$ for $\mathrm{l} \mathrm{h}$ to allow motile sperm to swim up. Aspirate supernatant into a clean test tube and centrifuge at $330 \times g$ for $7 \mathrm{~min}$. Aspirate final supernatant and resuspend sperm pellet in $0.5 \mathrm{~mL}$ of sperm wash media. Measure the final volume, and perform semen analysis on an aliquot of the sample (8).

7. Sperm preparation by density gradient: A double density gradient (lower density; 40-47\% "upper phase" and 80-90\% "lower phase") is used (8). Transfer $2.0 \mathrm{~mL}$ of the "lower phase" into a $15 \mathrm{~mL}$ conical centrifuge tube using a sterile pipette. Carefully add $2.0 \mathrm{~mL}$ of the "upper phase" to the top of the "lower phase." Place the liquefied semen sample (1-2 mL) on top of the "upper phase" layer and centrifuge at $330 \times g$ for $20 \mathrm{~min}$. Aspirate the upper and lower layers without disturbing the pellet. Add 2-3 mL of sperm wash media, and spin at $330 \times g$ for $7 \mathrm{~min}$. Remove the supernatant and resuspend the pellet in $1.0 \mathrm{~mL}$ of sperm wash media. Measure sperm count and motility in recovered fractions. Perform all steps at $37^{\circ} \mathrm{C}$.

8. This step must be performed in subdued light.

9. Example corrected ROS calculation:

Sperm Count $=12.6 \times 10^{6} / \mathrm{mL}$.

Patient average $\mathrm{ROS}=12,161 \mathrm{RLU} / \mathrm{s}$.

Negative control average $\mathrm{ROS}=8,850.3 \mathrm{RLU} / \mathrm{s}$.

Unadjusted $\mathrm{ROS}=12,161-8,850.3 \mathrm{RLU} / \mathrm{s}=3310.7 \mathrm{RLU} / \mathrm{s}$.

Adjusted $\mathrm{ROS}=3,310.7 / 12.6$.

$=262.7 \mathrm{RLU} / \mathrm{s} / 10^{6}$ sperm.

Result $=$ ROS positive.

10. In our lab, the criterion for acceptance is that control reads are $<20 \mathrm{RLU} / \mathrm{s} / 10^{6}$ sperm. Values $>20 \mathrm{RLU} / \mathrm{s} / 10^{6}$ sperm for 
controls are rejected and the assay is rerun. The reagent lot numbers and expiration dates are recorded.

11. The reconstituted vial is stable for 6 months when stored at $4^{\circ} \mathrm{C}$.

12. The reconstituted vial is stable for $24 \mathrm{~h}$ at $4^{\circ} \mathrm{C}$.

13. The reconstituted reagent is stable for 1 month when stored at $-20^{\circ} \mathrm{C}$.

14. The reconstituted vial is stable for $24 \mathrm{~h}$ at $4^{\circ} \mathrm{C}$.

15. The working solution is stable for $4 \mathrm{~h}$ at room temperature.

16. Label the vials for correct identification.

17. Any errors during pipetting can be highlighted on the template for any discrepancy in the final results.

18. A multichannel pipette should be used to pipette chromogen. Chromogen can be pipetted from a flat container.

19. This step should be completed as quickly as possible (within $1 \mathrm{~min}$ ). Hydrogen peroxide can be pipetted from a flat container using a multichannel pipette.

20. In addition the information can also be derived from the worksheet provided by the manufacturer and plugging each value in the spreadsheet available at www.caymanchem.com.

21. Reference ranges established in our lab are the following: Normal value: $>2,000 \mu \mathrm{M}$ Trolox, abnormal value: $<2,000 \mu \mathrm{M}$ Trolox. Since seminal OS results from an imbalance between ROS production and antioxidant defense, measurement of both ROS and TAC is important. It is essential to have a reproducible and reliable method for ROS measurement for clinical purposes. Strict quality control must also be taken into account for valid assessment in a clinical laboratory setting.

22. While measurement of ROS levels or TAC alone cannot precisely quantify OS, it is possible to combine these two parameters into one index score called ROS-TAC score (1). The ROS and TAC values from the controls can generate a reference scale of these two variables. In order to normalize values to the same distribution, $\log (\mathrm{ROS}+\mathrm{l})$ is used in calculations. The reason for this is that ROS levels if measured as $\times 10^{6} \mathrm{cpm} / 20$ million sperm can have a very wide range including zero, where no detectable ROS is generated. Therefore it is more accurate to convert these to log values and add a positive number such that the ROS value is always positive. Both TAC and $\log ($ ROS +1$)$ are initially standardized to $z$-scores so that both will have the same variability. These standardized scores are calculated by subtracting the mean values of the controls from the mean value of the patients, and dividing this number by the standard deviation of the control population. 
For details refer to our earlier study (1) where ROS values were expressed as cpm.

ROS-TAC score minimizes the variability of ROS and TAC alone. The ROS-TAC score reported by us earlier (1) was based on a group of normal healthy fertile men who had very low levels of ROS measured as $\times 10^{6} \mathrm{cpm} / 20$ million sperm. It is easy to convert cpm to RLU and vice versa $(10 \mathrm{RLU}=1 \mathrm{cpm})$.

The ROS-TAC score was found to be better than ROS or TAC alone in discriminating between fertile and infertile men. Infertile men with male factor infertility or an idiopathic diagnosis had significantly lower ROS-TAC scores than the healthy controls, and men with a male factor diagnosis who eventually were able to initiate a pregnancy had significantly higher ROSTAC scores than those who were not able to do so. The average ROS-TAC score for fertile healthy men was 50.0 \pm 10 , which was significantly higher $(p \pm 0.0002)$ than that of the infertile patients $(35.8 \pm 15)$. The probability of successful pregnancy was estimated at $<10 \%$ for values of ROS-TAC $<30$, but increased as the ROS-TAC score increased.Measurement of ROS in neat semen has proved to be an accurate and reliable test for assessing the OS status (6). Assessing ROS directly in neat semen also has diagnostic and prognostic capabilities identical to that of the ROS-TAC score. This approach overcomes the limitations of earlier methods where processing of semen could generate ROS by itself, thereby accurately representing the true in vivo OS status of an individual. While ROS levels were significantly lower in neat semen than in washed spermatozoa, ROS levels in neat semen demonstrated a strong positive correlation with ROS levels in washed semen $(9,10)$.

\section{References}

1. Sharma RK et al (1999) The reactive oxygen species-total antioxidant capacity score is a new measure of oxidative stress to predict male infertility. Hum Reprod 14: 2801-2807

2. Aitken RJ, Buckingham D (1992) Enhanced detection of reactive oxygen species produced by human spermatozoa with 7-dimethyl aminonaphthalin-1, 2-dicarbonic acid hydrazide. Int J Androl 15:211-219

3. Aitken RJ et al (1992) Reactive oxygen species and human spermatozoa: analysis of the cellular mechanisms involved in luminol- and lucigenindependent chemiluminescence. J Cell Physiol 151:466-477

4. Sharma RK, Agarwal A (1996) Role of reactive oxygen species in male infertility. Urology 48:835-850

5. Kobayashi H et al (2001) Quality control of reactive oxygen species measurement by luminol- dependent chemiluminescence assay. J Androl 22:568-574

6. Allamaneni SS et al (2005) Characterization of oxidative stress status by evaluation of reactive oxygen species levels in whole semen and isolated spermatozoa. Fertil Steril 83:800-803

7. Agarwal A et al (2004) Chemiluminescence technique for measuring reactive oxygen species. Reprod Biomed Online 9:466-468

8. Allamaneni SS et al (2005) Comparative study on density gradients and swim-up preparation techniques utilizing neat and cryopreserved spermatozoa. Asian J Androl 7:86-92

9. Saleh RA, Agarwal A (2002) Oxidative stress and male infertility: from research bench to clinical practice. J Androl 23:737-752

10. Saleh RA et al (2002) Leukocytospermia is associated with increased reactive oxygen species production by human spermatozoa. Fertil Steril 78:1215-1224 